\title{
Bilateral ectopic tubal pregnancy, following in vitro fertilisation (IVF)
}

\section{Tadeusz Issat ${ }^{1,3}$, Wojciech Grzybowski ${ }^{1}$, Artur J. Jakimiuk ${ }^{1,2}$}

\author{
${ }^{1}$ Department of Obstetrics and Gynecology Central Clinical Hospital of Ministry of Interior \\ and Administration, Warsaw, Poland \\ 2Department of Reproductive Health Research Institute of Mother and Child, Warsaw, Poland, \\ ${ }^{3}$ Department of Immunology, Center of Biostructure Research the Medical University of Warsaw, Poland,
}

\begin{abstract}
This is a case report of a 33 year-old-woman who underwent in vitro fertilization, because of primary infertility caused by fallopian tube factor. The patient underwent three trials of frozen embryo transfers (ET-CRYO). On the 26 ${ }^{\text {th }}$ day after the third probe of ET-CRYO she suffered from pelvic pain and vaginal bleeding. Transvaginal ultrasonographic scan revealed bilateral tubal ectopic pregnancy without fluid in the Douglas pouch and no gestational sac in the uterus. Laparoscopic bilateral salpingectomy was performed on the next day after admission. The postoperative course was uneventful.
\end{abstract}

Key words: ectopic tubal pregnancy, in vitro fertilisation

\section{Introduction}

The implantation of a trophoblast outside the uterus cavity occurs in $1.5 \%$ to $2 \%$ of all pregnancies. In more than $90 \%$, this occurs in the fallopian tubes, and 8 per 10 are located in the ampullar region [1]. According to the majority of reports the frequency of ectopic pregnancies has grown in the last 30 years [2].

However, bilateral tubal ectopic pregnancy is a rare clinical entity. It may occur in 1 per 200000 pregnancies $[3,4]$. We report a case of simultaneous bilateral tubal pregnancy that occurred in a patient submitted to IVF-ET, after the transfer of two frozen embryos.

\section{Case report}

A 33 year old woman gravida 1, para 1 with history of infertility caused by fallopian tube factor was admitted to our department complaining about pain and vaginal bleeding. In the past, i.e. 13 years ago she underwent the first laparoscopy, including salpingoplasty, abdominal cavity adhesiolysis and bilateral ovarian cysts removal. Four years later she was treated again by bilateral laparoscopic salpingotomy and salpingoplasty due to pyosalpinx. In 2004 the patient underwent the

Correspondence: J. Jakimiuk, Center for Reproductive Health, Institute of Mother and Child, Kasprzaka 17a, 01-211 Warsaw, Poland; tel.: (+4822) 3277033, e-mail: jakimiuk@yahoo.com third laparoscopy in order to carry out fallopian tubes plasty and adhesiolysis. In 2006 after in vitro fertilization the patient gave birth to an alive daughter after uncomplicated pregnancy and delivery. Since October 2008 the patient has been trying to get pregnant again with the help of IVF methods. The first two probes of two embryos transfer in October 2008 and January 2009 failed. The patient was admitted to our department on the $26^{\text {th }}$ day after the third probe of two embryos transfer. Pelvic pain sustained for 24 hours. At the admission the patient did not display peritoneal symptoms. Her general health condition was good, including respiratory and circulatory functions. In gynecological examination vaginal portion of cervix was found to be cylindrical with round opening and clear cervical disk. Vagina contained blood-tinted secretion. The corpus of uterus was insignificantly enlarged, movable and painless. Left and right adnexa were enlarged, bilaterally palpable, with palpable pain. Laboratory tests revealed elevated levels serum of $\beta$-hCG at $6327 \mathrm{mIU} / \mathrm{Ml}$ as well as progesterone at $205.5 \mathrm{nmol} / \mathrm{L}$.

Ultrasonographic scan revealed AP uterus size of $53.7 \mathrm{~mm}$, and empty uterine cavity Endometrium was homogenous, evenly thick ( $10.6 \mathrm{~mm}$, i.e. double layer). The left fallopian tube was thickened, of the size $24 \times 19 \mathrm{~mm}$ with an embryo (Crown Rump Length (CRL) $4.8 \mathrm{~mm}$ ). The left ovary was regular, of $25 \times 20$ $\mathrm{mm}$. The right fallopian tube was thickened, of the size 
$22 \times 25 \mathrm{~mm}$ also with an embryo inside (CRL $3.8 \mathrm{~mm}$ ). The right ovary was regular, of $34 \times 26 \mathrm{~mm}$. FHR of both embryos was not observed. There was no fluid in the pouch of Douglas. Due to the above-mentioned circumstances the decision of surgical treatment was taken.

Laparoscopy revealed the corpus of the uterus to be of regular size, to the right fallopian tube insignificantly thickened in the area of fimbriae, to the left livedo and fallopian tube thickened in the isthmus. Left and right ovaries were intacted macroscopically. Fallopian tubes were removed bilaterally. Postoperative period was uncomplicated. Serum $\beta$-hCG level decreased on the second day after the surgery to $450 \mathrm{mIU} / \mathrm{Ml}$. On the third day following the surgery the patient was discharged from the hospital in a good condition.

\section{Discussion}

The first pregnancy obtained with IVF by Steptoe at al. was ectopic, in a patient with history of tubal factor infertility [5]. Theories regarding the genesis of spontaneous ectopic pregnancy are well known. Damage to the endosalpinx produced by sexually transmitted infections, distortion of pelvic anatomy caused by disease like endometriosis or by adhesions after previous interventions as appendectomy, surgery performed in the fallopian tubes, mullerian malformations, hormone concentration imbalance during the menstrual cycle, late ovulations, history of a previous ectopic pregnancy etc. [1]. In literature there were almost 50 cases of bilateral ectopic pregnancy since 1997, and almost $50 \%$ of them were reported as spontaneous bilateral ectopic pregnancies [1]. The first case of a bilateral tubal ectopic pregnancy after IVF-ET was published in 1983 [6], but spontaneous bilateral tubal pregnancy was reported in 1918 [7]. Three theories have been proposed to explain the origin of a bilateral ectopic pregnancy after IVF. The first, the embryos might be directly injected into the tubes because of a deficient transfer technique. The second hypothesis is that, the embryos correctly transferred to the endometrial cavity could regressively migrate to the tubes as a consequence of endometrial secretions that push them in that direction. The third theory is the so-called "spray" effect produced during transfer; when emptying the hypodermic syringe, the embryos could possibly "be pushed" toward the tubal portions. However, in properly functioning tubes embryos should return to the endometrial cavity. When they are dysfunctional or damaged, implantation outside the eutopic endometrium can occur more frequently [1,7].

The diagnosis of ectopic pregnancy should always be considered in patients undergoing IVF-ET, because of its increased incidence compared to natural conception. Although the incidence of a bilateral ectopic pregnancy is not very high, and it may also occur after spontaneous pregnancy, both adnexa should be examined when diagnosis of an ectopic pregnancy is made. As in this case, early diagnosis is essential for the prevention of significant maternal morbidity and mortality.

Acknowledgements: Tadeusz Issat is a recipient of a START stipend from the Foundation for Polish Science

\section{References}

[ 1] De Los Ríos JF, Castaneda JD, Miryam A. Bilateral ectopic pregnancy. J Minim Invasive Gynecol. 2007;14(4):419-27.

[2] Stabile I, Grudzinskas JG. Ectopic pregnancy: a review of incidence, etiology and diagnostic aspects. Obstet Gynecol Surv. 1990;45(6):335-47.

[ 3] Jřnler M, Rasmussen KL, Lundorff P. Coexistence of bilateral tubal and intrauterine pregnancy. Acta Obstet Gynecol Scand. 1995;74(9):750-2.

[4] Stewart HL Jr. Bilateral ectopic pregnancy. West J Surg Obstet Gynecol. 1950;58(11):648-56.

[5] Steptoe PC, Edwards RG. Reimplantation of a human embryo with subsequent tubal pregnancy. Lancet. 1976;1(7965):880-2.

[ 6] Trotnow S, Al-Hasani S, Hünlich T, Schill WB. Bilateral tubal pregnancy following in vitro fertilization and embryo transfer. Arch Gynecol. 1983;234(1):75-8.

[ 7] Hewitt J, Martin R, Steptoe PC, Rowland GF, Webster J. Bilateral tubal ectopic pregnancy following in-vitro fertilization and embryo replacement. Case report. $\mathrm{Br} J$ Obstet Gynaecol. 1985;92(8):850-2. 